6 Dastur HM. Diagnosis and neurosurgical treatment of tuberculous diseases of CNS. Neurosurg Rev 1983;6:111-9. 7 Citow JS, Ammirati $M$. Intramedullary tuberculoma of spinal cord: case report. Neurosurgery 1994;35:327-30.
8 Bhatoe HS. Intramedullary spinal cord tuberculoma-case report. Indian f Tuberculosis 1996;43:99-100.

9 Rich AR, McCordick HA. Pathogenesis of tuberculous meningitis. Bull fohns Hopkins Hosp 1933;52:5-37.

\title{
Pyrexia of unknown origin
}

\author{
L Thia, Y E Nakhuda
}

A 32-year-old Indian man presented with a 4-week history of intermittent pyrexia with rigours, associated with malaise and weight loss. Physical examination was normal except for pyrexia without evidence of lymphadenopathy. The white cell count was $13.2 \times 10^{9} / 1(3.5-11.0)$ with increased lymphocytes. Erythrocyte sedimentation rate and C-reactive protein were elevated. Liver function tests showed mildly raised liver enzymes. Blood and sputum cultures were negative for acid-fast bacilli. A computed tomography (CT) scan of the abdomen revealed a multiloculated mass in the head of the pancreas with peripancreatic and mesenteric lymphadenopathy (figure).

The Royal Liverpool University Hospitals, Departments of Surgery and Radiology, Prescot Street, Liverpool L7 8XP, UK L Thia

Y E Nakhuda

Correspondence to Dr Lena Thia, 88 Langham Court, Wyke Road, West

Wimbledon, London SW 20 $8 R R$, UK

Accepted 25 November 1998

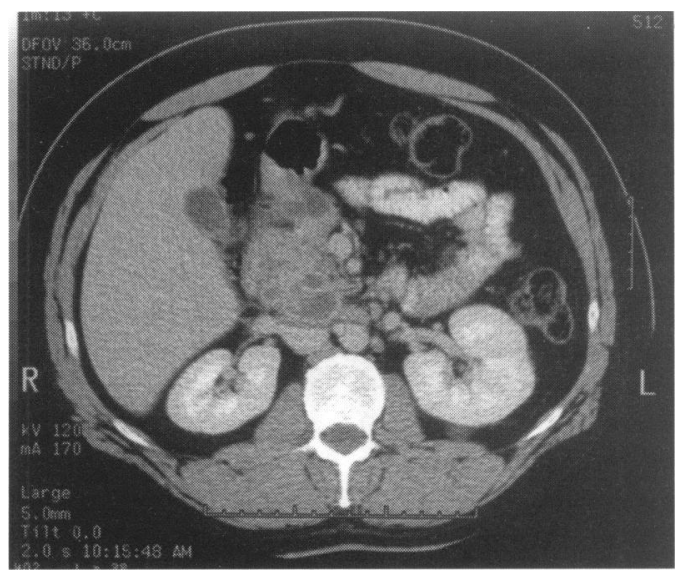

\section{Questions}

1 Suggest the most likely differential diagnosis in this case.

2 What further investigations should be performed?
Figure CT scan of the abdomen showing an enlarged head of the pancreas with focal hypodense lesions 


\section{Answers}

QUESTION 1

Pyrexia with a mass in the head of pancreas can be due to three general categories of disease process (box). Bacterial and viral infections can cause recurrent septicaemia. Tuberculosis is still a major cause of pyrexia of unknown origin (PUO) in developing countries and among Asian patients in the UK. Pancreatic abscess secondary to cholangitis or infected phlegmon/ pseudocyst from pancreatitis can also cause PUO with a mass in the head of pancreas. Lymphoma frequently produce a prolonged period of fever before other manifestations emerge. Primary lymphoma represents less than $1 \%$ of pancreatic neoplasms. Secondary lymphomas are more common. Mass lesions involving the pancreas may also represent extension of disease from neighbouring organs: carcinoma of the duodenum, ampulla of Vater, bile duct, and gall bladder, as well as gastric neoplasms and lymph node metastases around the pancreas, may be indistinguishable from a primary pancreatic neoplasm.

\section{QUESTION 2}

In view of the mildly deranged liver function tests and CT findings, an endoscopic retrograde cholangiopancreaticography was performed, which revealed an abnormal orifice in the second part of the wall of the duodenum. The patient went on to have a CT-guided aspiration and needle biopsy of this pancreatic mass. Histological and microbiological assessments were inconclusive. Finally our patient underwent an open cholecystectomy and surgical biopsy of the pancreatic mass to obtain adequate tissues for diagnostic smears and cultures.

\section{Discussion}

Surgical exploration revealed a mass in the pancreatic head, associated with nodal masses in the proximity of the small bowel mesentery and a small shrunken gall bladder. Smears from the specimen were positive for acid- and alcohol-fast bacilli on special fluorescence stain. Histological assessment showed epitheloid granulomas, giant cells and caseation necrosis. Cultures of excised tissue were positive for Mycobacterium tuberculosis.

Tuberculosis of the pancreas is a rare clinical entity even in countries in which abdominal and pulmonary tuberculosis is common. ${ }^{12}$ It

1 Crowson MC, Perry M, Burden E. Tuberculosis of pancreas. A rare cause of obstructive jaundice. $\mathrm{Br} \mathcal{F}$ Sur 1984;71:239.

2 Bhansali SK. Abdominal tuberculosis. Experience with 300 cases. Am f Gastroenterol 1977;67:324-37.

3 Varshney S, Johnson CD. Tuberculosis of the pancreas. Postgrad Med 7 1996;80:564-6.

\section{Causes of mass in head of pancreas}

- infections (bacterial, viral, tuberculosis)

- inflammations (infected phlegmon/pseudocyst, cholecystitis)

- neoplasia (adenocarcinoma, islet cell tumour, mucinous and serous cystic tumour, metastasis (from melanoma, lungs, breast, ovarian), direct extension of tumour, lymphoma

may manifest itself in various ways such as anorexia, malaise, low-grade fever, weight loss, night sweats, pancreatic mass or abscess, obstructive jaundice, pancreatitis and even malignancy. Due to varied presenting features and the rarity of pancreatic tuberculosis, the differentiation of a granulomatous lesion forming an isolated pancreatic mass from the more common malignant lesion may be difficult, hence often requiring an exploratory laparotomy with biopsy. ${ }^{3}$ The diagnosis of pancreatic tuberculosis is most often not suspected prior to laparotomy unless there is evidence of tuberculosis elsewhere, especially in the lungs. Our patient had no prior history of pulmonary tuberculosis.

Literature on radiological findings in pancreatic tuberculosis is scant. ${ }^{4} \mathrm{CT}$ and ultrasound may show an enlarged pancreas with focal hypodense or hypo-echoic lesions, usually in the head region, which are nonspecific. Other ancillary findings are peripancreatic lymph nodes, mesentery and mural thickening, lesions in other solid viscera and ascites. CT-guided aspiration or ultrasound-guided biopsy of a pancreatic mass can be diagnostic if granulomatous inflammation is noted and one proceeds to acid-fast staining that may document the bacilli, although negative bacteriologic staining is common in abdominal tuberculosis. ${ }^{5}$ In conclusion, primary pancreatic tuberculosis should be considered as a diagnostic possibility in patients presenting with fever who show focal intrapancreatic lesions on imaging.

\section{Final diagnosis}

Primary pancreatic tuberculosis.

Keywords: pyrexia of unknown origin; tuberculosis; pancreatic mass

4 Takhtani D, Gupta S, Suman K, Kakkar N, et al. Radiolog of pancreatic tuberculosis: a report of three cases. $A m \mathcal{F}$ Gas troenterol 1996;91:1832-4.

5 Haddard FS, Ghossain A, Sawaya E, Nelson AR. Abdominal tuberculosis. Dis Colon Rectum 1987;30:724-35. 